\title{
Noninvasive Ventilation Is Interrupted Frequently and Mostly Used at Night in the Pediatric Intensive Care Unit
}

\author{
Katherine R Schlosser, Gaston A Fiore, Craig D Smallwood, John F Griffin, Alon Geva, \\ Mauricio Santillana, and John H Arnold
}

\begin{abstract}
BACKGROUND: Noninvasive ventilation (NIV) is commonly used to support children with respiratory failure, but detailed patterns of real-world use are lacking. The aim of our study was to describe use patterns of NIV via electronic medical record (EMR) data. METHODS: We performed a retrospective electronic chart review in a tertiary care pediatric ICU in the United States. Subjects admitted to the pediatric ICU from 2014 to 2017 who were mechanically ventilated were included in the study. RESULTS: The median number of discrete device episodes, defined as a time on support without interruption, was 20 (interquartile range [IQR] 8-49) per subject. The median duration of bi-level positive airway pressure (BPAP) support prior to interruption was $6.3 \mathrm{~h}$ (IQR 2.4-10.4); the median duration of CPAP was $6 \mathrm{~h}$ (IQR 2.1-10.4). Interruptions to BPAP had a median duration of $6.3 \mathrm{~h}$ (IQR 2-15.5); interruptions to CPAP had a median duration of $8.6 \mathrm{~h}$ (IQR 2.2-16.8). Use of NIV followed a diurnal pattern, with $44 \%$ of BPAP and $42 \%$ of CPAP subjects initiating support between 7:00 PM and midnight, and 49\% of BPAP and 46\% of CPAP subjects stopping support between 5:00 AM and 10:00 AM. CONCLUSIONS: NIV was frequently interrupted, and initiation and discontinuation of NIV follows a diurnal pattern. Use of EMR data collected for routine clinical care allowed the analysis of granular details of typical use patterns. Understanding NIV use patterns may be particularly important to understanding the burden of pediatric ICU bed utilization for nocturnal NIV. To our knowledge, this is the first study to examine in detail the use of pediatric NIV and to define diurnal use and frequent interruptions to support. Key words: noninvasive ventilation; mechanical ventilation; electronic health records; child; intensive care. [Respir Care 2020;65(3):341-346. (C) 2020 Daedalus Enterprises]
\end{abstract}

\section{Introduction}

Use of noninvasive ventilation (NIV) is widespread ${ }^{1-4}$ and has increased in pediatric ICUs across the world. ${ }^{5}$ Most of the work describing the increased use of NIV has focused upon the timing of NIV in relation to intubation ${ }^{6-8}$

Drs Schlosser, Smallwood, Geva, and Arnold, and Mr Griffin are affiliated with the Division of Critical Care Medicine, Department of Anesthesiology, Critical Care and Pain Medicine, Boston Children's Hospital, Boston, Massachusetts. Dr Schlosser is also affiliated with the Division of Pediatric Critical Care, Department of Pediatrics, Columbia University Irving Medical Center, New York. Dr Geva, Dr Santillana, and Mr Fiore are affiliated with the Computational Health Informatics Program, Boston Children's Hospital, Boston, Massachusetts.

Dr Geva was supported by National Institutes of Health/National Institute of Child Health and Human Development grant T32-HD-040128. The other authors have disclosed no conflicts of interest. or its utility for pediatric acute respiratory distress. ${ }^{9}$ NIV has been applied and studied for a number of years in the adult population, ${ }^{10-14}$ yet there are few adult or pediatric data sets describing the details of its usage. Use patterns such as duration of uninterrupted support, duration of interruptions, and proportion of patients supported continuously versus only overnight are not well reported in the literature. In addition, frequency of changes to NIV support affects staff workload; thus, as NIV is used with

\footnotetext{
Dr Schlosser presented a version of this paper at the Society of Critical Care Medicine Annual Congress, held February 25-28, 2018, in San Antonio, Texas.

Correspondence: Katherine R Schlosser MD, 3959 Broadway, CHN-10 24, New York, NY 10032. E-mail: ks3629@cumc.columbia.edu.
}

DOI: $10.4187 /$ respcare.06883 
increasing frequency, ideal staffing patterns may be affected. One way to gather data about NIV use is through registries, but this limits the volume and type of data that can be collected. ${ }^{15}$ Therefore, we sought to describe use patterns of NIV in a large tertiary care pediatric ICU using a technique of extracting data from routine charting in the electronic medical record (EMR).

Specifically, this study had 3 main aims. The first 2 aims were clinical: to quantify nocturnal use of NIV in a pediatric ICU, and to quantify interruptions to NIV use in a pediatric ICU. The third aim of this study was to demonstrate a data science-focused approach to extracting this information from the EMR without requiring a manual, labor-intensive, prospective study.

\section{Methods}

We performed a single-center retrospective cohort study at Boston Children's Hospital in Boston, Massachusetts. We included subjects admitted to the pediatric ICU from 2014 to 2017 who received invasive mechanical ventilation at any point. Demographic and clinical data were extracted from the institutional data warehouse. At our institution, the data warehouse captures clinical, financial, and operational data gathered from multiple data sources, including the EMR. Data chosen for analysis included primary fields in the EMR where respiratory support was documented by clinicians. Only structured data-entry fields were analyzed; free-text fields were excluded (Fig. 1). Diagnostic categories were determined from the primary discharge diagnostic code. This study was approved by the Boston Children's Hospital institutional review board.

Invasive mechanical ventilation was defined as a patient receiving support through an endotracheal tube. Patients who received support via tracheostomy were excluded from this study. NIV refers to subjects receiving CPAP or bi-level positive airway pressure (BPAP) through a full face mask or nasal interface. The oxygen therapy group included subjects receiving any other respiratory support not included in the 2 categories already described (eg, nasal cannula, high-flow nasal cannula, aerosol mask). Room air refers to subjects without any respiratory support devices.

Use of NIV varies from unit to unit in terms of who makes decisions about changes. In the unit where this study occurred, NIV is initiated in a multidisciplinary fashion with input from physician, nursing, and respiratory therapy team members. Escalation of delivered pressure often also involves a multidisciplinary discussion, and the physician team is involved. Weaning of pressure is often done by respiratory therapists at the bedside following institutional protocols. Titration of oxygen delivered is often done by nursing at the bedside following institutional protocols and targets established on bedside rounds.

\section{QUICK LOOK}

\section{Current knowledge}

Use of noninvasive ventilation (NIV) to support pediatric patients with acute respiratory failure has been increasing. Many pediatric patients requiring NIV are supported in pediatric ICUs. In the setting of widespread electronic medical record adoption, researchers have been increasingly looking to data science to improve efficiency of clinical knowledge generation.

\section{What this paper contributes to our knowledge}

The use of NIV followed a diurnal pattern with increased use at night compared to during the day. Additionally, NIV was frequently interrupted, with support lasting on average about $6 \mathrm{~h}$ prior to interruption. This study demonstrates a data science-focused approach to extracting this information from the electronic medical record without requiring a manual, labor-intensive, prospective study.

Breaks from support either occur as part of planned weaning protocols discussed on bedside rounds with the multidisciplinary team or by nursing and respiratory therapy as part of routine care such as suctioning, bathing, oral hygiene, patient tolerance, and skin care.

Current documentation practices in the EMR vary widely, and these computerized systems provide multiple locations for data regarding respiratory support to be captured. Even within an EMR system, individual provider documentation demonstrates variability. As a consequence, we sought to create a strategy to identify initiation and discontinuation of respiratory support, with multiple layers of redundancy that would account for variation in documentation.

Because initiation and discontinuation times of NIV are not consistently recorded in the EMR explicitly as structured data, we constructed a rules-based algorithm to determine start and stop times from other EMR data. We identified 3 main fields where respiratory support was documented: oxygen $\left(\mathrm{F}_{\mathrm{IO}_{2}}\right)$ delivery device, oxygen $(\mathrm{L} / \mathrm{min})$ delivery device, and oxygen source. Each field has dropdown list of values to choose among, including an option for Other with manual entry by a clinician. We then assigned a hierarchy with the field allowing most choices for support type documentation as primary (ie, oxygen $\left(\mathrm{F}_{\mathrm{IO}_{2}}\right)$ delivery device), with the other two following sequentially (ie, oxygen $(\mathrm{L} / \mathrm{min})$ delivery device, and then oxygen source). Any time when multiple conflicting respiratory support types were documented was flagged. We also used supporting fields to assess for endotracheal tube versus 


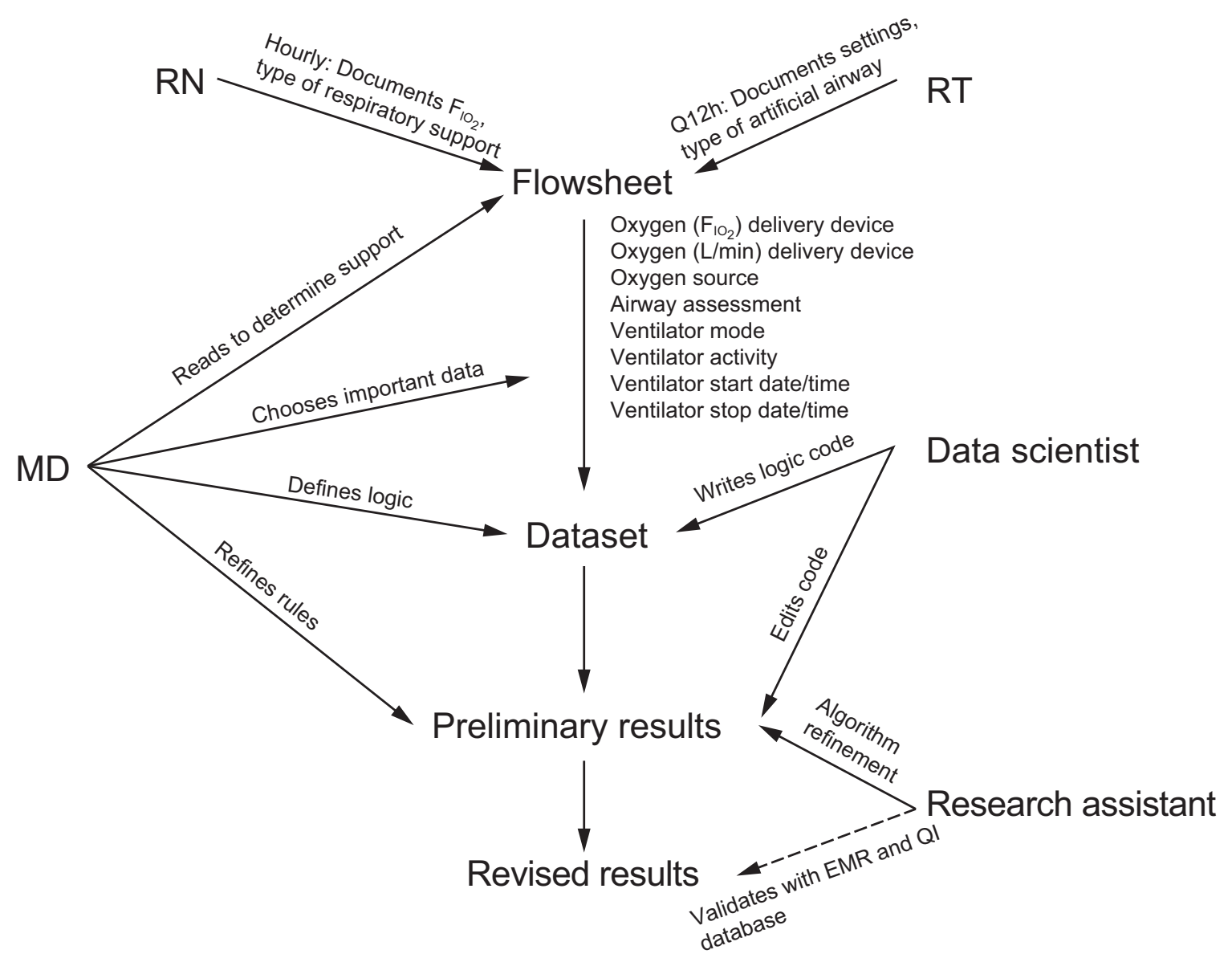

Fig. 1. Flow diagram of data extraction and processing. $\mathrm{RN}=$ registered nurse; $\mathrm{RT}=$ respiratory therapist; $\mathrm{MD}=$ physician; $\mathrm{Q} 12 \mathrm{~h}=\mathrm{every}$ $12 \mathrm{~h} ; \mathrm{EMR}=$ electronic medical record; QI = quality improvement.

tracheostomy tube and mode of ventilation. We defined a support duration as the time between first and last documentation of a support type where no other intervening modes were documented. Instances where a support type wass documented only once did not allow duration to be defined, but it did interrupt the previous support type. Gaps in documentation of $>24 \mathrm{~h}$ were recorded as a support stop. We performed iterative refinement of this algorithm using manual review of the EMR as the accepted standard. To validate our approach, a study member (JG) not involved with algorithm development independently determined start and stop times for respiratory support based on review of the EMR for a sample of subjects.

We analyzed all respiratory support per subject across all pediatric ICU admissions. Because the respiratory support data were not normally distributed across our population, we reported continuous parameters as median values (interquartile range [IQR]). The EMR-based algorithm was implemented using Python 3 (Python Software Foundation, Wilmington, Delaware).

\section{Results}

There were 2,302 subjects who met clinical inclusion criteria. The primary diagnostic categories of these subjects are shown in Table 1. Of these subjects, 1,449 had sufficient data for analysis. The median total duration of NIV was 57.7 h (IQR 19.0-192.0); duration of other support is shown in Table 2. The median number of discrete device episodes, defined as a time on support without interruption, was 20 (IQR 8-49) per subject.

When looking at support utilization across all pediatric ICU stays during the study period, the median duration of support prior to interruption was similar for BPAP at $6.3 \mathrm{~h}$ (IQR 2.4-10.4) and for CPAP at $6 \mathrm{~h}$ (IQR 2.110.4). The interruptions to BPAP had a median duration of $6.3 \mathrm{~h}$ (IQR 2-15.5), and interruptions to CPAP had a median duration of $8.6 \mathrm{~h}$ (IQR 2.2-16.8) (Fig. 2). Duration off NIV had a bimodal pattern with peaks at 2-3 $\mathrm{h}$ and around $15 \mathrm{~h}$. Use of NIV followed a diurnal pattern, with $44 \%$ of subjects receiving BPAP and $42 \%$ of subjects receiving CPAP initiating support between 
7:00 PM and midnight; $49 \%$ of subjects receiving BPAP and $46 \%$ of subjects receiving CPAP stopped support between 5:00 AM and 10:00 AM (Fig. 3).

In addition to performing statistical analyses on the data, we developed graphical representations of respiratory support use for clinical or quality purposes. One example, demonstrated in Figure 4, is a representation of a single subject's use of respiratory support across their ICU stay.

Table 1. Subject Characteristics

\begin{tabular}{lc}
\hline \hline Age, y & $6.1(1.4-14.4)$ \\
Male & $844(58)$ \\
Primary Diagnosis & \\
$\quad$ Neurologic & $330(23)$ \\
Surgery & $308(21)$ \\
Respiratory & $301(21)$ \\
Other & $241(17)$ \\
Infectious & $130(9)$ \\
Hematologic/oncologic & $114(8)$ \\
Cardiac & $33(2)$
\end{tabular}

$\overline{\text { Data are presented as }} n(\%)$ or mean (interquartile range).

Table 2. Duration of Respiratory Support

\begin{tabular}{lcc}
\hline \hline & Subjects, $n$ & $\begin{array}{c}\text { Median (IQR) } \\
\text { Total Duration, h }\end{array}$ \\
\hline $\begin{array}{l}\text { Noninvasive ventilation } \\
\text { Oxygen therapy }\end{array}$ & 591 & $57.7(19.0-192.0)$ \\
\hline & 917 & $39.3(6.7-367.7)$ \\
\hline CPAP & Episodes, & Median (IQR) \\
BPAP & 3,423 & Hours per Episode \\
\hline
\end{tabular}
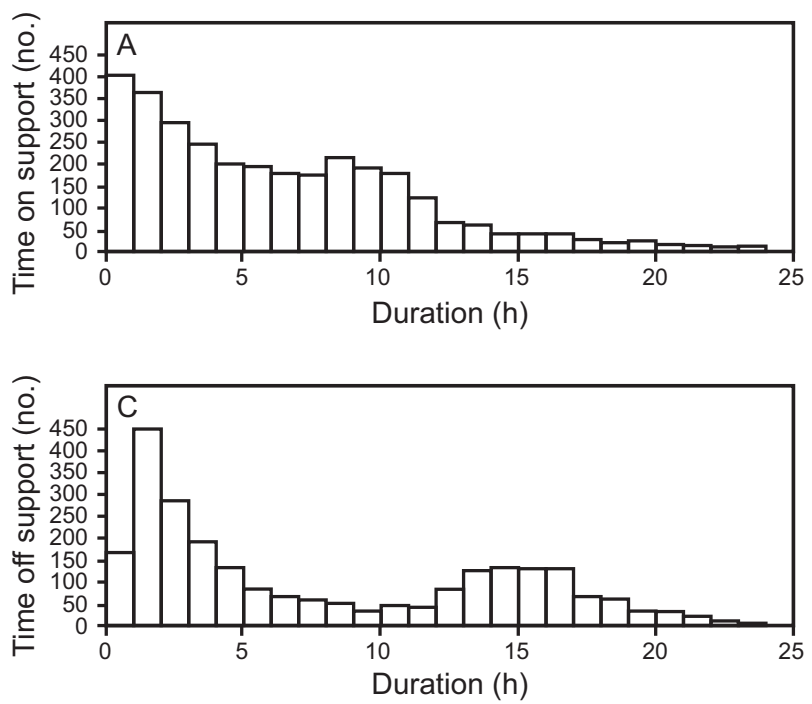

Fig. 2. Interruptions of noninvasive ventilation. CPAP is shown in panels A and C; BPAP is shown in panels B and D. The top panels present the duration on respiratory support without interruption, and the lower panels present the duration of interruptions.
This representation highlights that the subject was extubated to BPAP, and then weaned off BPAP slowly, eventually settling into nighttime use of BPAP with daytime hours spent on room air.

\section{Discussion}

Our study indicates that NIV is interrupted frequently and that NIV has a diurnal pattern to its use. To our knowledge this is the first study to examine in detail the use of pediatric NIV. We propose that future studies on NIV use in pediatric populations gather data at a granular level so that NIV use patterns can be linked to outcomes. This study did not categorize interruptions into different categories such as planned weaning trials, breaks for comfort or routine care, or interruptions for suctioning or respiratory interventions. Now that we have demonstrated that interruptions are frequent, it would be beneficial to track categories of interruptions in future studies. Additionally, duration of use and interruptions to use are only a small part of the data that could be captured and analyzed about NIV use, including physiologic parameters, alarms, and settings. We propose incorporating all of these variables into future studies looking at outcomes.

While we propose a solution that works with only existing EMR documentation as a feasible and efficient manner of extracting this information, improving documentation of breaks, including reasons for breaks, would certainly benefit future researchers as well.

In this study, we were able to quantify the diurnal use of NIV. While many pediatric and adult patients use nighttime NIV at home, ${ }^{16}$ this poses a specific challenge in pediatric hospitals. ${ }^{917}$ In the hospital setting, where pa-
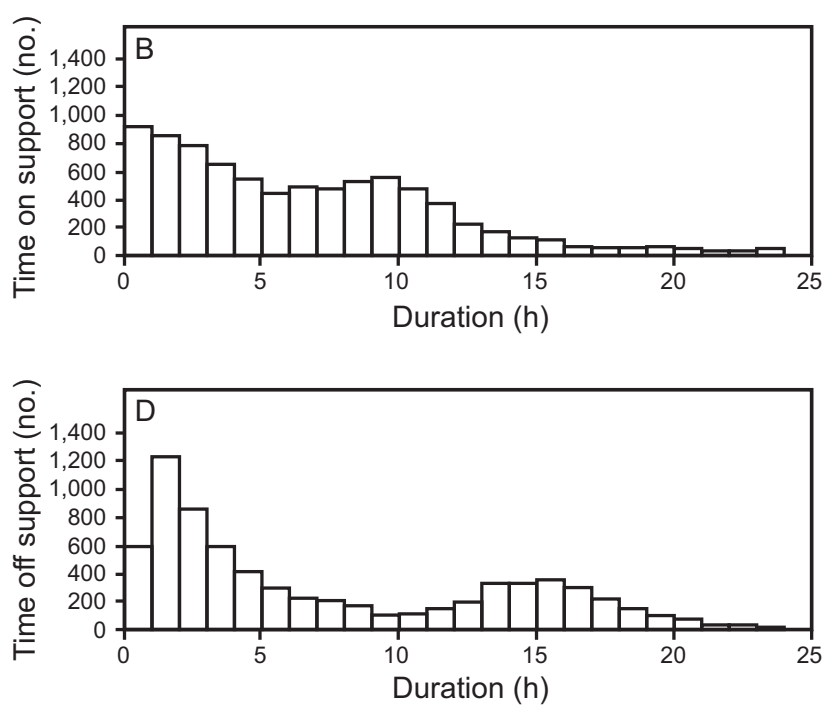

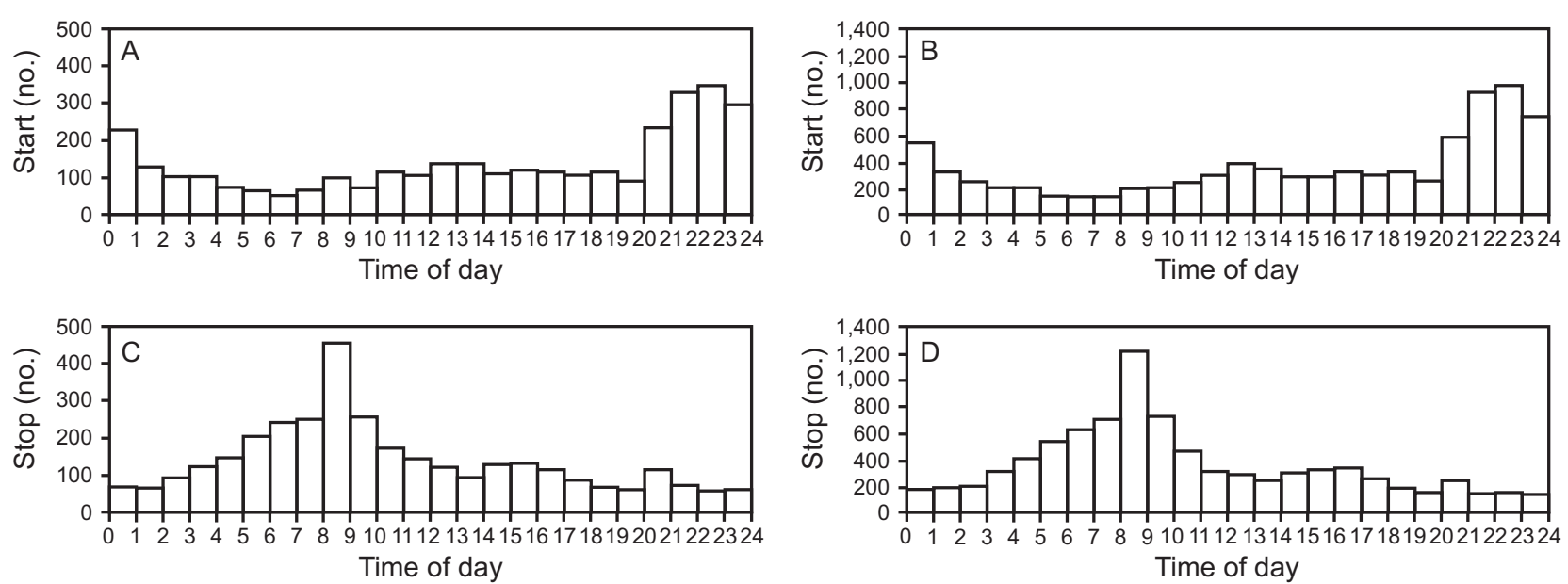

Fig. 3. Diurnal use of noninvasive ventilation (NIV). CPAP is shown in panels A and C; BPAP is shown in panels B and D. Time of day is shown on the $x$ axis, with both 0 and 24 representing midnight. The $y$ axis shows the number of occurrences of NIV being started or stopped that occurred during each hour of the day. Panels $A$ and $B$ show the time at which respiratory support was started; panels $C$ and D show the time at which respiratory support was stopped or interrupted.

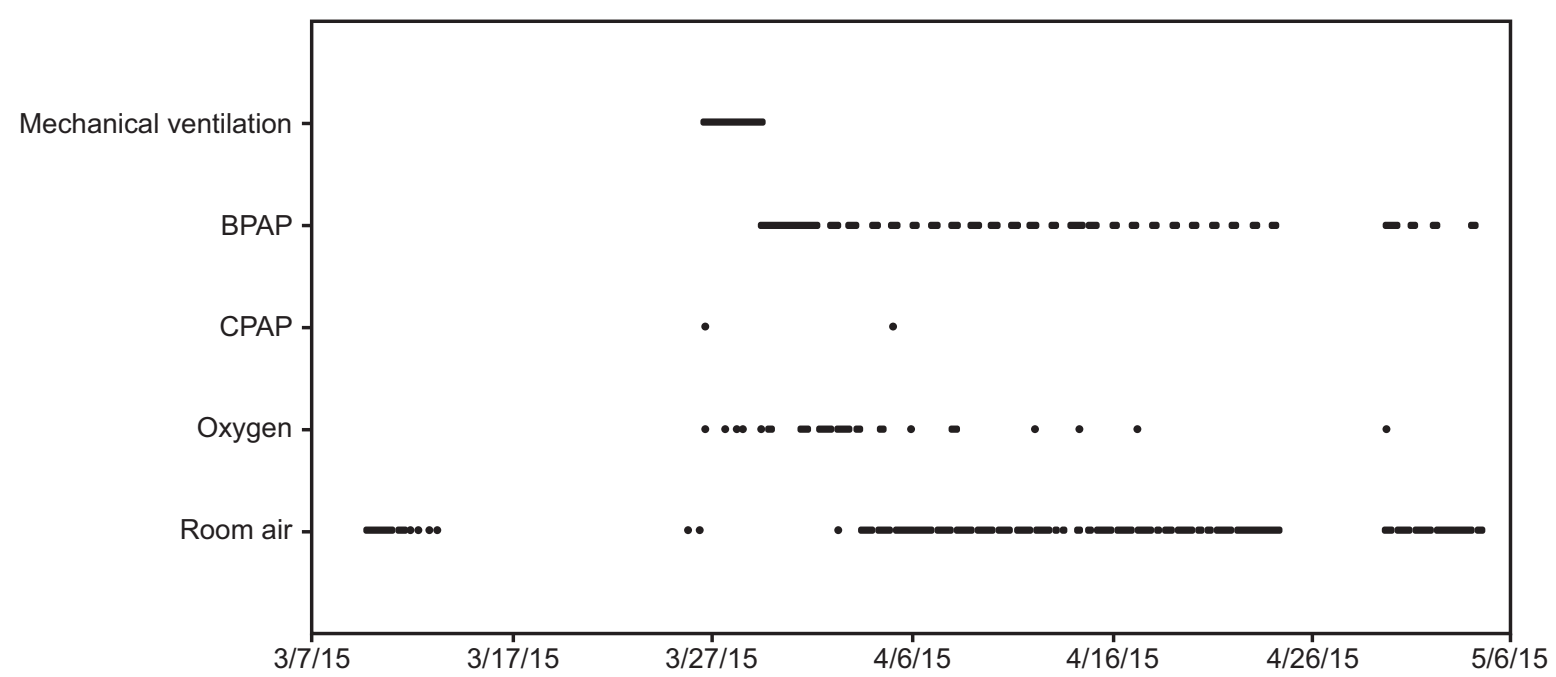

Fig. 4. Graphical representation of a single subject's respiratory support over time.

tients may have an infection or may be recovering from surgery, they are not at their physiologic baseline. Additionally, if a parent or guardian is not at the bedside, pediatric patients may be unable to safely call for help or remove their own mask. Understanding the burden of pediatric ICU bed utilization for nocturnal NIV use may allow creative staffing models, such as a dedicated respiratory therapist for nighttime NIV, to make pediatric ICU beds available while maintaining patient safety and monitoring. ${ }^{3}$ This work also highlights the work burden created by multiple device initiations and terminations around a traditional 7:00 AM or 7:00 PM change of shift.

This study has several limitations. The first is that it was performed at a single center, and our population may not reflect that of all pediatric ICUs across the country. Our approach of partnering with data scientists to extract raw data from the EMR and define respiratory support initiation and discontinuation through a series of logic-based rules allows this method to be replicated across centers to define their own mechanically ventilated populations on the basis of EMR data. A second limitation is that we have not yet directly linked duration of NIV or interruptions to NIV to patient outcomes. Duration of mechanical ventilation has been described as an important marker of patient outcomes. ${ }^{18,19}$ The association of NIV use with outcomes in pediatric patients is an area of ongoing research. Our results suggest that such research ought to account for interruptions to NIV use and weaning patterns in any such outcomes-oriented studies. Additionally, understanding granular use patterns of pediatric respiratory support has 


\section{NIV IN THE PEDIATRIC ICU}

implications beyond patient outcomes, including staffing models and bed utilization. Further work is needed to directly measure the impact on staff care burden of these respiratory support patterns.

\section{Conclusions}

This study demonstrates the feasibility of understanding respiratory support utilization at a granular level. Future research will focus upon linking duration of NIV and interruptions in NIV to patient outcomes. We recommend future studies investigating the use of NIV gather data about use patterns so that a better understanding of NIV use can be developed and linked to patient outcomes.

\section{ACKNOWLEDGMENTS}

We thank Jonathan Bickel MD MSc and the Business Intelligence and Clinical Research Informatics team for their assistance with data extraction.

\section{REFERENCES}

1. Fanning JJ, Lee KJ, Bragg DS, Gedeit RG. U.S. attitudes and perceived practice for noninvasive ventilation in pediatric acute respiratory failure. Pediatr Crit Care Med 2011;12(5):e187-e194.

2. Romans RA, Schwartz SM, Costello JM, Chanani NK, Prodhan P, Gazit AZ, et al. Epidemiology of noninvasive ventilation in pediatric cardiac ICUs. Pediatr Crit Care Med 2017;18(10):949-957.

3. Fedor KL. Noninvasive respiratory support in infants and children. Respir Care 2017;62(6):699-717.

4. Emeriaud G, Essouri S, Tucci M. Noninvasive ventilation in the PICU: one step closer. Crit Care Med 2017;45(6):1103-1104.

5. Wolfler A, Calderini E, Iannella E, Conti G, Biban P, Dolcini A, et al. Evolution of noninvasive mechanical ventilation use: a cohort study among Italian PICUs. Pediatr Crit Care Med 2015;16(5):418427.

6. Morris JV, Ramnarayan P, Parslow RC, Fleming SJ. Outcomes for children receiving noninvasive ventilation as the first-line mode of mechanical ventilation at intensive care admission: a propensity scorematched cohort study. Crit Care Med 2017;45(6):1045-1053.
7. Yaman A, Kendirli T, Odek C, Ates C, Tasyapar N, Gunes M, et al. Efficacy of noninvasive mechanical ventilation in prevention of intubation and reintubation in the pediatric intensive care unit. J Crit Care 2016;32:175-181.

8. Crulli B, Loron G, Nishisaki A, Harrington K, Essouri S, Emeriaud G. Safety of paediatric tracheal intubation after non-invasive ventilation failure. Pediatr Pulmonol 2016;51(2):165-172.

9. Essouri S, Carroll C, Pediatric Acute Lung Injury Consensus Conference Group. Noninvasive support and ventilation for pediatric acute respiratory distress syndrome: proceedings from the Pediatric Acute Lung Injury Consensus Conference. Pediatr Crit Care Med 2015;16(5 Suppl 1):S102-S110.

10. Cabrini L, Landoni G, Oriani A, Plumari VP, Nobile L, Greco M, et al. Noninvasive ventilation and survival in acute care settings: a comprehensive systematic review and metaanalysis of randomized controlled trials. Crit Care Med 2015;43(4):880-888.

11. Nava $\mathrm{S}$, Hill $\mathrm{N}$. Non-invasive ventilation in acute respiratory failure. Lancet 2009;374(9685):250-259.

12. Hess DR. Noninvasive ventilation for acute respiratory failure. Respir Care 2013;58(6):950-972.

13. Burns KE, Meade MO, Premji A, Adhikari NK. Noninvasive ventilation as a weaning strategy for mechanical ventilation in adults with respiratory failure: a Cochrane systematic review. CMAJ 2014; 186(3):E112-E122.

14. Keenan SP, Sinuff T, Burns KE, Muscedere J, Kutsogiannis J, Mehta $\mathrm{S}$, et al. Clinical practice guidelines for the use of noninvasive positive-pressure ventilation and noninvasive continuous positive airway pressure in the acute care setting. CMAJ 2011;183(3):E195E214.

15. Ducharme-Crevier L, Essouri S, Emeriaud G. Noninvasive ventilation in pediatric intensive care: from a promising to an established therapy, but for whom, when, why, and how? Pediatr Crit Care Med 2015;16(5):481-482.

16. Amin R, Al-Saleh S, Narang I. Domiciliary noninvasive positive airway pressure therapy in children. Pediatr Pulmonol 2016;51(4): 335-348.

17. Cummings JJ, Polin RA, Committee on Fetus and Newborn. Noninvasive respiratory support. Pediatrics 2016;137(1):e20153758.

18. Fraser J, Henrichsen T, Mok Q, Tasker RC. Prolonged mechanical ventilation as a consequence of acute illness. Arch Dis Child 1998; 78(3):253-256.

19. Hill AD, Fowler RA, Burns KE, Rose L, Pinto RL, Scales DC. Long-term outcomes and health care utilization after prolonged mechanical ventilation. Ann Am Thorac Soc 2017;14(3):355-362. 